\title{
Experimental Study of Vibrating Screen Beam Based on Power Flow Method
}

\author{
Bingxian $\mathrm{Ou}^{1, \mathrm{a}}$, Junxia Yan ${ }^{2, \mathrm{~b}}$ \\ ${ }^{1}$ Jiangsu province special equipment safety supervision inspection institute of Wuxi Branch, Wuxi \\ 214174, China \\ ${ }^{2}$ School of Mechanical Engineering, Jiangnan University, Wuxi 214122, China \\ aobxobx@163.com, baixia0122@163.com
}

Keywords: Vibrating screen, redundant constraints beam, power flow, experimental study.

\begin{abstract}
In order to study the problem of beam crack and side plate tearing bring by large-scale and high-speed development of vibrating screen, redundant constraints beam is designed by means of adding redundant constraints to the beam, and vibration experiment system is put up. Input power flow formula of system is derived based on cross-spectral density method proposed by Fahy, the input power flow of improved and original beams is measured, and the input power flow variation with frequency is analyzed. It obtained that, amplitude of input power flow of redundant constraints beam appeared in low frequency, attenuation in high frequency is fast, and at about $100 \mathrm{~Hz}$ it tends to balance; amplitude of input power flow of original beam appeared in high frequency, attenuation in high frequency is slow, and $150 \sim 200 \mathrm{~Hz}$ it tends to balance. The results showed that, redundant constraints beam has inhibition and attenuation of vibration energy, it beneficial to realize high-speed of vibrating screen.
\end{abstract}

\section{Introduction}

With the constant improvement of the national economy and productivity, the development of the vibrating screen tends to large-scale, high speed and light-duty. Many production instance tells us that the beam of the screen and the life of the side panel drops greatly with large scale and quite speed [1-3]. Thus satisfy the vibrating screen with high screening efficiency and large capacity at the same time, how to guarantee the structural strength and stiffness to become the key to achieve large-scale and high speed of the vibrating screen [4-6].

Redundant constraint structure widely used in industry, construction and bridge engineering, and other fields because of the structure with redundant constraints can still bear the load after the redundant constraints are destroyed. From protection against sudden destruction, redundant constraint structure has stronger defense capabilities than statically determinate structure. And it provides the feasibility to design the beam of vibrating screen with redundant constraint structure [7].

In this paper, the beam of linear vibrating screen is improved for redundant constraints. Through the experiment, measure the input power flow of the beam structure before and after the improvement.

\section{Experimental system}

Experimental model. According to the geometric similarity, dynamic similarity, movement similarity, boundary conditions and so on, experimental model of vibrating screen is developed as shown in figure 2, the beam structure choose square steel tube (hereinafter referred to as illustrated) with length of $1 \mathrm{~m}$, cross section size of $110 \mathrm{~mm}$ x $75 \mathrm{~mm}$ and thickness of $5 \mathrm{~mm}$. For redundant constraint structure, the improved beams with four square steel tube through the cross board connection, single side tube cross section size is $40 \mathrm{~mm}$ x $25 \mathrm{~mm}$, thickness is $3 \mathrm{~mm}$, as shown in figure 1 . The design of redundant constraint structure beam is based on mechanics of materials, and 
according to the principle of equal quality and moment of inertia in order to not increase the screen body quality and has the same bending stiffness of the beam before improvement [8].

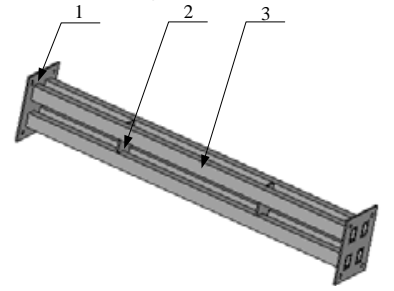

(a) Redundant constraints structure beam

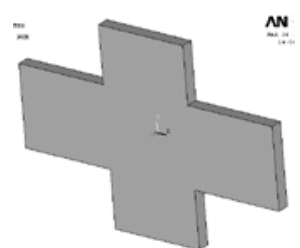

(b) cross board

1-flange 2-cross board 3- square steel tube

Fig.1 Redundant constraints structure beam

Experimental equipment. There is a homemade vibration experiment system(as shown in figure 2) with a vibrating screen, two vibration motor, a frequency modulator, and a vibration analysis system including an intelligent signal acquisition instrument, combined anti mixed filter amplifier, four piezoelectric acceleration sensors, a high elastic capacity hammer, and intelligent data acquisition and signal analysis software.
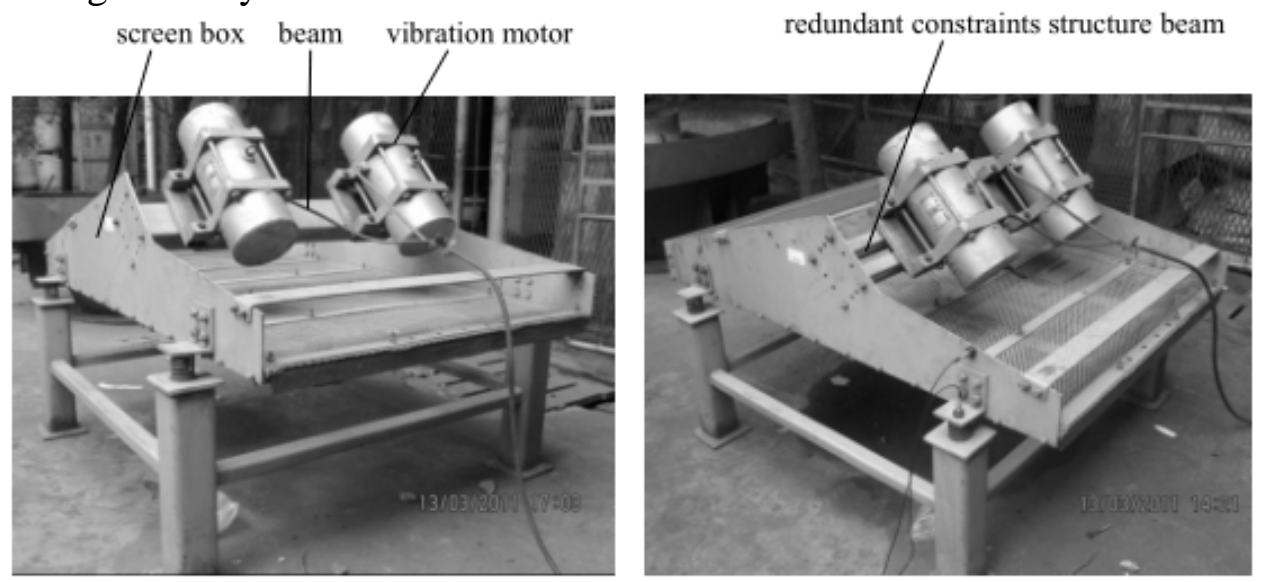

Fig.2 Vibration experiment system

\section{Power flow measurement}

Method of measurement. In this experiment it adopts the cross spectral method [9] to calculate the input power flow through measuring the signal of the exciting force and the acceleration signal of the input point.

The input power flow is represented as follows:

$P_{\text {input }}=\lim _{T \rightarrow \infty} \frac{1}{2 \pi} \int_{-T}^{T} f(t) v(t) d t=\lim _{T \rightarrow 0, \tau \rightarrow 0} \frac{1}{2 \pi} \int_{-T}^{T} f(t) v(t-\tau) d t=R(f, v, 0)$

$f(t)$-----force signal of force sensor connect hammer;

$a(t)$-----acceleration signal of acceleration sensor;

$v(t)$-----unknown speed signal;

$R(f, v, \tau)$------correlation function of force signal and speed signal;

$G(f, a, \omega)$------power spectral density function of force signal and acceleration signal;

$G(f, v, \omega)$------ power spectral density function of force signal and speed signal.

After derivation

$P_{\text {in }}=\frac{1}{\omega} \operatorname{Im}[G(f, a, \omega)]=\frac{2 h}{\omega N} \operatorname{Im}\left[f^{*}(\omega) \cdot a(\omega)\right]$

T-----acquisition signal cycle; N-----collection points; h-----sampling interval, $h=T / N$; 
$f^{*}(\omega)$------complex conjugate of $f(t)$ after Fourier transform in the time domain; $a(\omega)$----- Fourier transform of $a(t)$.

Measure $f(t)$ and $a(t)$ through impedance, cross power spectral density is obtained after Fourier transform. And plug in (2) the input power flow can be calculated.

Experiment and result analysis. Measure the input power flow in 9 equal division place of the beam respectively. Collect force and acceleration signal by hammer the division place in illustrated tube and redundant constraints beam. Export the measured data and calculate, the change of input power flow with frequency is obtained. As in figure 3 shown, (a) (g) respectively express the input power flow contrast of illustrated tube and redundant constraint beam in the corresponding measuring position.

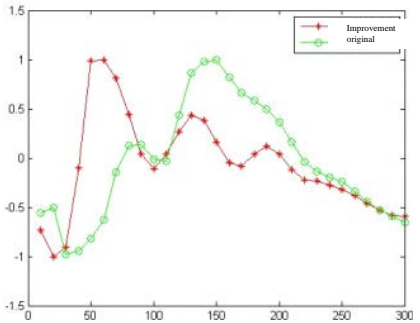

(a)

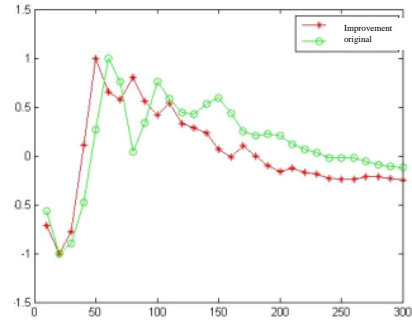

(d)

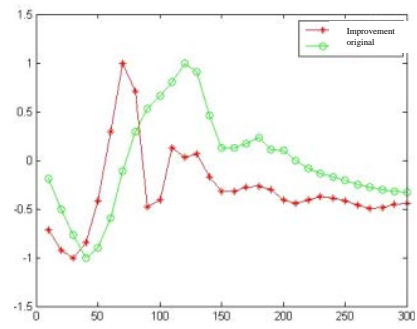

(h)

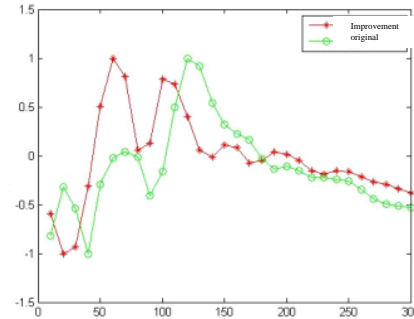

(b)

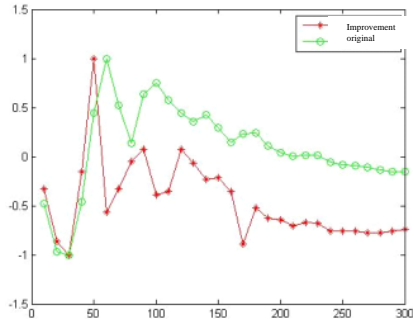

(e)

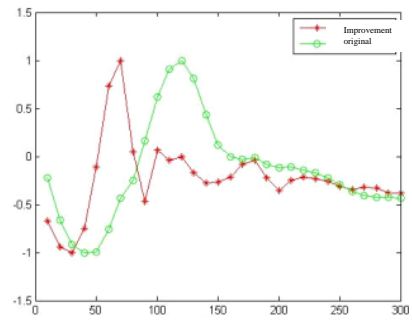

(i)

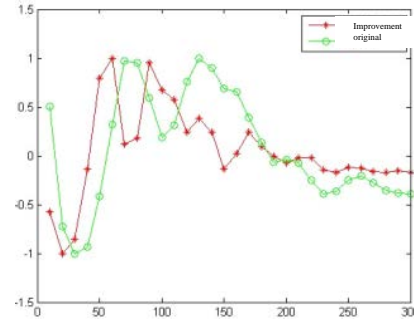

(c)

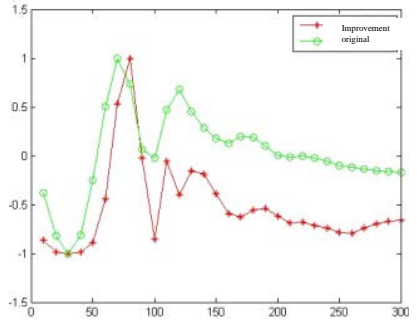

(f)

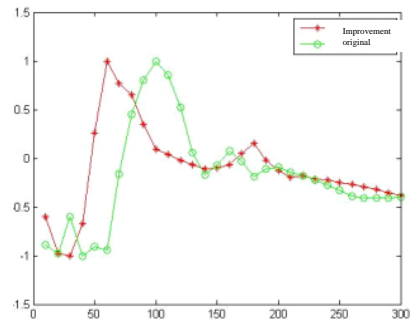

(g)

Fig.3 Input power flow variation with frequency

Normalization processing is made for exciting force input and input power flow calculated It is not hard to see from figure above, no matter in which measurement point, input power flow of redundant constraints beam has more fluctuation on high frequency than illustrated tube, and the amplitude appear on low frequency, at $100 \mathrm{~Hz}$ or so approaches equilibrium. Input power flow amplitude of illustrated tube move to middle-frequency, at $150 \sim 200 \mathrm{~Hz}$ approaches equilibrium, and the curve always on top of redundant constraint beams. Based on the principle of system energy conservation, the input power flow is sum of passed to the system and system attenuation consumption, so under the same condition, the more the faster attenuation, the less power flow passed to the system, which can explain redundant constraint beam has the effect of energy inhibition and attenuation.

\section{Conclusion}

By adding constraints to improve beam vibrating screen for redundant constraint structure. Analyze the input power flow along with the change of frequency using the test system of vibration. Concluded that for improved redundant constraint beam amplitude of input power flow appears in 
low frequency, attenuation soon in high frequency, and at about $100 \mathrm{~Hz}$ approaches equilibrium, which explain cross board added can suppress and attenuate the vibration energy.

\section{References}

[1] HE Xiao-mei, LIU Chu-sheng, ZHANG Cheng-yong. Dynamic optimal design of large vibrating screen based on an augmented lagrangian method [J]. Journal of China University of Mining \& Technology, 2009, 38(1):80-85.

[2] YAN Xue-min, CHEN Qing-ru. Modelling study of the constructional strength of vibration screen [J]. Journal of China University of Mining \& Technology, 1989, 18(3):18-24.

[3] LIU Chu-sheng, JIANG Xiao-wei, ZHANG Shi-min, et al. Calculation and experimental verification of vibrating direction angle of varying trajectory banana screen [J]. Journal of China University of Mining \& Technology, 2011, 40(5):737-724.

[4] LIU Chu-sheng, LU Jin-xin. Nonlinear characteristic analysis of screening rod of elastic-rod vibrating screen [J]. Journal of China University of Mining \&Technology, 2009, 38(4):476-480.

[5] ZHAO La-la, LIU Chu-sheng, YAN Jun-xia, et al. Numerical simulation of particles flow on the vibrating screen plate using a 3D discrete element method [J]. Journal of China University of Mining \&Technology, 2010, 39(3):414-419.

[6]JIAO Hong-guang, ZHAO Yue-min, LUO Zhen-fu, et al. Parameters optimization of probability screen plane [J]. Journal of China University of Mining \&Technology, 2006, 35(3):384-388.

[7] LI Qing-lu, YANG Jing-ning. Optimal design of the constant strength static-indeterminate beam under multi-loading [J]. Journal of Gansu sciences, 2009, 21(4):93-96.

[8] YAN Jun-xia. Basic study on the dynamic characteristics and reliability of large vibrating screen with high-speed [D]. Xuzhou: China University of Mining \&Technology, 2011.

[9] F J Fahy. Measurement of mechanical input power to a structure [J]. Journal of Sound and Vibration, 1969, 10(3):517-518.

[10] ZHU Xiang. Vibrational power flow characteristics of cracked structures and the damage detection [D]. Wuhan: Huazhong University of science \& technology, 2007.

[11] ZHANG Wen-de, LIU Xi-jun, XIAO Long-xiang. Estimating phase difference of two vibration signals with common frequency using cross- spectral density function [J]. Mechanics in Engineering, 2003, 25(1):58-59. 